\title{
Analyze students' learning processes \\ to innovate university teaching. \\ The Formative Educational Evaluation model \\ of the University of Bologna
}

\section{Analizzare i processi di apprendimento degli studenti per innovare la didattica universitaria. II modello di Formative Educational Evaluation dell'Università di Bologna \\ Nicoletta Balzaretti, Elena Luppi, Dina Guglielmi, Ira Vannini ${ }^{1}$}

\begin{abstract}
The article aims to highlight how the knowledge of university students' characteristics is a key-point for planning innovative teaching. The Bologna University Project refers to a Formative Educational Evaluation model, which is focused on higher education teaching skills and aims to have an impact on teaching and learning processes' multiple levels and dimensions, in a diachronic perspective. The application of the model to different academic contexts of the University of Bologna was carried on by two evaluation research pilot studies with "Nursing" and "Economics and Finance" (CLEF) Degree teachers. This model includes evaluation actions specifically oriented in the formative sense (analysis and progressive re-planning of teaching actions supported by ad hoc higher education teachers training interventions) in order to promote teachers' capacity for reflection, self-analysis of their teaching practices and specific training actions based on innovative methodologies.
\end{abstract}

Keywords: Higher Education, formative educational evaluation, teaching quality, pilot study.

\section{Premessa}

Il Processo di Bologna del 1999, volto ad armonizzare i vari sistemi europei di higher education verso un'organizzazione tale da garantire la trasparenza e leggibilità dei percorsi formativi e dei titoli di studio, ha comportato un rinno-

${ }^{1}$ Le Autrici afferiscono all'Alma Mater Studiorum Università di Bologna.

I $\S \S 1,6,6.1$. e 6.2 sono stati scritti da Nicoletta Balzaretti; i $\S \S 2$ e 2.1 da Ira Vannini; i $\S$ $3,4,4.1,5,5.1$ e 7 da Elena Luppi; il $\S 6.3$ da Dina Guglielmi. Tutto l'impianto del contributo è frutto di una riflessione comune tra tutte le Autrici. 
vamento nei curricula dei sistemi accademici europei, a cui è corrisposta la traduzione degli obiettivi formativi dei corsi di studi in termini di risultati di apprendimento (learning outcomes) e di profili di competenze. Questo passaggio ha cambiato sostanzialmente gli scenari pedagogici del mondo dell'istruzione superiore: tradizionalmente impostati su una logica dell'«apprendimento per conoscenze e contenuti», i vari attori si sono e si stanno ancora interrogando su come favorire, supportare e mettere in atto strategie organizzative e didattiche verso un «apprendimento per competenze» intese come entità complesse, nelle quali si integrano molteplici elementi, quali innanzitutto le conoscenze, insieme poi ad abilità e aspetti metacognitivi, competenze trasversali strategiche per l'apprendimento e per la vita (Le Boterf, 1990; Pellerey, 1983, 2004). In questo senso, costruire e aumentare il proprio livello di competenza significa essere in grado di mobilizzare (in maniera sempre più efficace) $i$ vari elementi al fine di utilizzarli in modo pertinente all'interno di specifici contesti.

Se la logica accademica "tradizionale" prevedeva un curriculum descrittivo di ciò che lo studente doveva conoscere, comprendere ed essere in grado di dimostrare alla fine di un percorso formativo (moduli o insieme di moduli di insegnamento), un profilo per competenze chiama in causa la finalità di ogni corso di studio e rappresenta una combinazione di competenze specifiche di area disciplinare e competenze trasversali intese come una sinergia di attributi cognitivi e metacognitivi, attitudini interpersonali, intellettuali, pratiche e valori etici che ne connotano il profilo professionale in uscita.

I corsi di studio universitari non possono, nell'attuale contesto politico-istituzionale, essere pensati come un insieme di moduli di insegnamento scarsamente in connessione tra loro; l'istanza è quella di una organicità di saperi che possano orientare lo sviluppo autonomo, da parte degli studenti, di apprendimenti significativi per le loro vite professionali e personali. Questa prospettiva richiede un forte coordinamento a livello accademico dei diversi attori che intervengono nel processo di apprendimento: dal management universitario, in termini di politiche, strategie e servizi amministrativi di supporto; al personale docente, chiamato a concepire i propri insegnamenti con modalità di supervisione, assessment e innovazione nelle risorse; agli studenti e alle studentesse, veri protagonisti del proprio imparare (Serbati e Zaggia, 2012). Questi ultimi - e le loro metacompetenze connesse all'apprendimento universitario - sono oggetto di ricerca e riflessione del presente contributo: due ricerche pilota nell'Ateneo bolognese portano alla luce metodologie e risultati che sottolineano l'importanza di conoscere, come variabile costituente della progettazione della didattica universitaria, le caratteristiche degli studenti legate alle competenze di tipo metacognitivo ovvero alle strategie di apprendimento e ad alcune dimensioni motivazionali ed affettive che regolano l'essere studenti in contesti accademici. 


\section{Un modello di ricerca valutativa in contesti accademici}

Creare le condizioni affinché la didattica universitaria possa efficacemente promuovere competenze è dunque un'esigenza di grande importanza per il sistema universitario, in particolare laddove si desideri il più possibile spostare l'asse di equilibrio da una logica improntata sulla mera selezione verso logiche di promozione di competenze e di innalzamento del livello intellettuale di tutti gli studenti o della maggior parte di essi. L'attenzione si focalizza su un'innovazione dei processi di insegnamento-apprendimento nel contesto universitario che sia efficacemente orientata a sviluppare elevate competenze per gli studenti, in modo diffuso. Ciò comporta affrontare il problema, altrettanto ampio e articolato, di quali siano le condizioni e gli aspetti che rendono una didattica universitaria realmente efficace.

Pur nell'impossibilità di definire in modo univoco, in questo contributo, l'ampio e complesso concetto di qualità nella didattica (cfr. Harvey e Green, 1993; Harvey e Williams, 2010), consideriamo come uno standard da perseguire un approccio ai processi di insegnamento e apprendimento che comporti l'attivazione di strategie di mediazione didattica effettivamente idonee allo sviluppo delle competenze di tutti gli studenti, con attenzione alle loro diverse caratteristiche e abilità in ingresso, ovvero agli elementi cognitivi del processo di apprendimento e a quelli metacognitivi (motivazione e volizione, abilità strategiche e di problem solving, percezione di sé e stili attributivi, ecc.), che agiscono sullo sviluppo delle competenze scientifiche e disciplinari.

Lavorare per una didattica di tal genere, si è detto, è questione complessa che chiama in causa sia la necessità di supportare le competenze didattiche di docenti e ricercatori universitari sia l'esigenza di creare condizioni istituzionali facilitanti la qualità dei processi di insegnamento.

\section{La Formative Educational Evaluation come riferimento teorico-metodologico}

Ma quale approccio metodologico può essere di supporto per intervenire nei contesti universitari e sostenere i colleghi docenti nell'acquisizione di adeguate competenze didattiche e nella presa di decisioni coerenti per il miglioramento dei percorsi formativi?

L'interrogativo che ci siamo posti come gruppo di ricerca interdisciplinare di Scienze dell'Educazione mirava a individuare un modello metodologico di riferimento capace di garantire a gruppi di docenti universitari (impegnati didatticamente su uno stesso curricolo formativo) una costante disponibilità di informazioni valide sui loro studenti e sui processi di insegnamento-apprendimento in cui erano coinvolti, utili a innescare confronti collegiali efficaci per 
la presa di decisioni e per l'individuazione di espliciti bisogni formativi. La risposta che abbiamo individuato - utilizzata finora in due progetti pilota dell'Ateneo bolognese ${ }^{2}-\grave{e}$ rintracciabile nel campo dell'educational evaluation research (cfr. Ryan, Bradley Cousins, 2009), un ambito complesso, per sua natura interdisciplinare e connesso al paradigma cosiddetto del pragmatismo (cfr. Mertens, 1998, 2018; Greene, 2008), le cui radici più profonde sono state rintracciate da Murphy (1990) e da Cherryholmes (1992) in Dewey. Questa prospettiva è capace di far dialogare costantemente, pur se in modo ampiamente problematico e sempre orientato da questioni valoriali (Walberg e Haertel, 1990; Becchi e Bondioli, 1994; Husen e Postlethwaite, 1994; Scriven, 1999; Kellaghan e Stufflebeam, 2003), l'esigenza della misurazione (della raccolta di dati validi e affidabili) con l'urgenza di assumere decisioni e implementare ipotesi di soluzione nei contesti valutati. Si tratta certamente di un campo complesso, non semplice da proporre (almeno dal punto di vista teorico) a gruppi di docenti universitari, ma certamente utile quando - insieme a colleghi di altre discipline - se ne fa esperienza diretta all'interno di specifiche realtà contestuali. Nell'esperienza bolognese si è scelto di fare riferimento a uno specifico filone della ricerca valutativa, quello della formative educational evaluation, nel suo più specifico significato di un processo valutativo che impegna costantemente tutti soggetti coinvolti in un lavoro di analisi dettagliata dei dati e di ricostruzione dei percorsi sottoposti a valutazione. La formative evaluation è infatti ricorsivamente incentrata su momenti di analisi dell'esistente e di ri-costruzione dei percorsi in vista del miglioramento (Vertecchi, 1976), essa consente di mettere in campo abilità diagnostiche, decisionali, progettuali dei soggetti e - così facendo - consente l'apprendimento e la crescita degli stessi soggetti coinvolti (cfr. Guasti, 2013).

Impegnarsi in percorsi di formative educational evaluation significa inoltre impegnarsi in processi di negoziazione, democraticamente ed eticamente orientati (House e Howe, 2003), nei quali ciascuno ha l'opportunità di partecipare alla presa di decisioni collettive in vista del miglioramento e nei quali ciascuno stakeholder ha la possibilità di confrontarsi e cooperare per la definizione delle strategie operative da mettere in campo, anche traendone giovamento in termini crescita professionale personale. Ciò che infatti trasversalmente emerge è che una valutazione così intesa porta anche con sé potenzialità di crescita e sviluppo, dei soggetti e dei contesti nei quali i processi si danno.

Un tale approccio metodologico di riferimento è rappresentabile come un processo ciclico in cui fasi di misurazione, confronto, riflessione, presa di decisioni e riprogettazione sono via via proposte e riproposte a un gruppo di lavoro che - nel nostro caso - ha coinvolto sia i docenti universitari interessati sia i ri-

\footnotetext{
${ }^{2}$ Ulteriori due progetti sono attualmente iniziati con i colleghi dei Corsi di studio in Veterinaria e in Ingegneria Offshore.
} 
cercatori, che hanno svolto varie funzioni nei momenti della rilevazione dei dati, dell'analisi, del supporto alla collegialità, della formazione laddove si individuavano carenze nelle competenze dei docenti.

La Figura 1 sintetizza la dinamica delle fasi di lavoro di questo approccio basato sulla formative educational evaluation; nello specifico, la prima direzione di lavoro riguarda la rilevazione e analisi dei dati, quale momento di raccolta sistemica, attraverso indicatori validi e procedure rigorose, di diverse tipologie di informazioni per lo studio del contesto e dei processi in atto; la seconda fase è quella della restituzione dei dati e della conseguente individuazione dei bisogni formativi dei docenti universitari, momento centrale poiché focalizzato sulla condivisione e la promozione di processi di autovalutazione e riflessività degli attori coinvolti; la terza fase è relativa a ipotesi di riprogettazione didattica e miglioramento intesa come momento operativo-decisionale in cui gli attori del contesto universitario ipotizzano collegialmente possibili azioni e direzioni di cambiamento. In questa fase sono inseriti anche specifici interventi formativi rivolti ai colleghi docenti, in risposta ai bisogni individuati.

Fig. 1 - Approccio metodologico di formative educational evaluation, rielaborato ed applicato agli studi pilota nell'Ateneo bolognese (Betti, Vannini, 213; Betti, Davila et al., 2015)

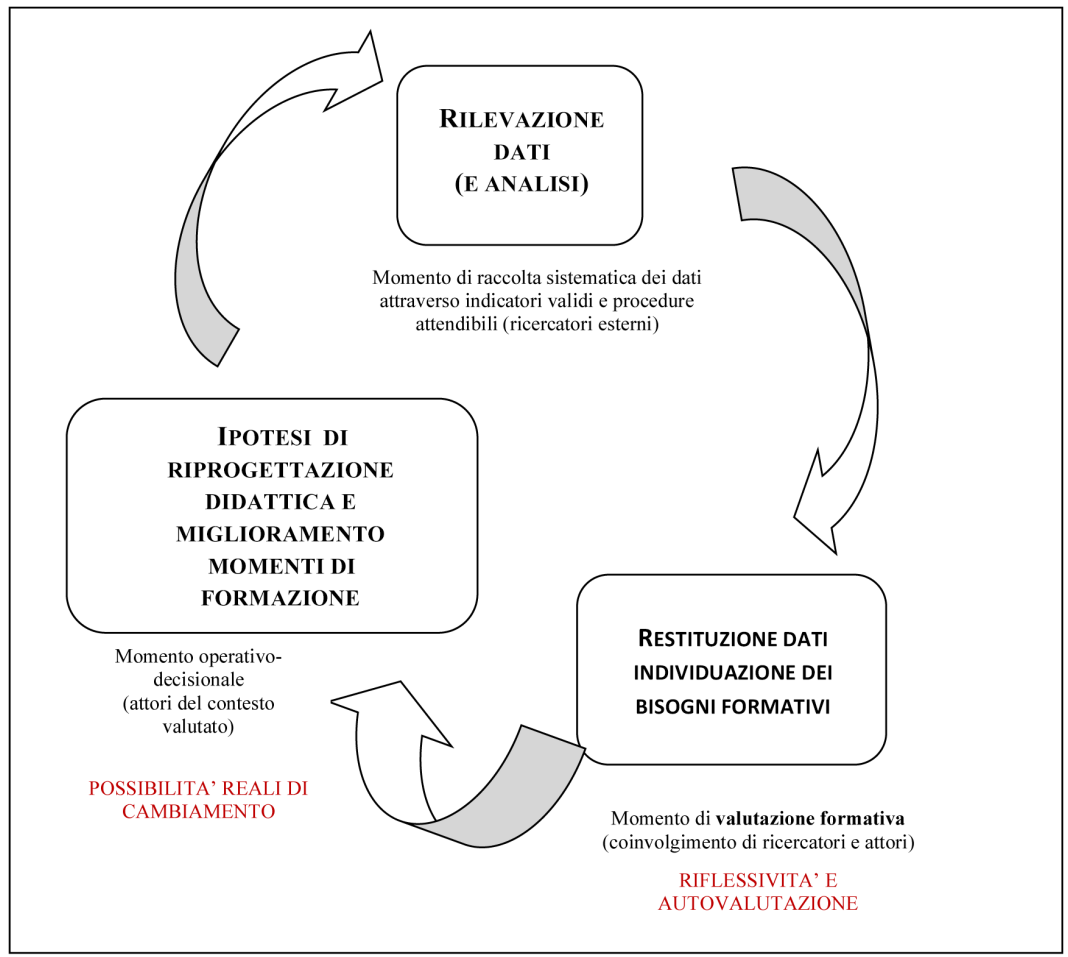




\section{La messa a fuoco sulle caratteristiche degli studenti}

Come si è visto, all'interno di un approccio metodologico di formative educational evaluation, una particolare attenzione è focalizzata sulla raccolta, in fase di "rilevazione dati", di numerose e diversificate dimensioni, in primis delle caratteristiche degli studenti in termini di livelli di padronanza nelle competenze trasversali ovvero nelle strategie per l'apprendimento in ingresso e in uscita dal percorso universitario.

In questo contributo, ci focalizzeremo in particolare su questa specifica fase, al fine di osservarne le potenzialità in termini di ricaduta sul miglioramento dei processi didattici.

La conoscenza del profilo di apprendimento degli studenti universitari è stato il punto di partenza, in due studi pilota dell'Ateneo bolognese, realizzati nei corsi di studio (CdS) triennali in Infermieristica e in Economics and Finance; l'obiettivo, coerentemente col modello di formative educational evaluation, era quello di attivare una riflessione collegiale sia sulla progettazione didattica dei corsi universitari sia sulla rilevazione di quelle che docenti e figure professionali ritengono debbano essere le abilità/capacità "in ingresso" dello studente prerequisiti per affrontare il corso di studi - e le competenze da implementare nel corso del percorso accademico.

La centralità degli studenti e delle studentesse, dei loro saperi e delle competenze che la formazione universitaria può promuovere in loro è un principio che l'Associazione Europea delle Università (EUA) (Gebel e Zhang, 2018; Amundsen e Haakstad, 2017; ESU, 2015) pone al centro delle linee di indirizzo per l'innovazione delle pratiche didattiche e l'innalzamento della qualità dell'Accademia. A partire da un confronto con le istituzioni educative europee, nell'ambito della European Higher Education Area (EHEA), la EUA propone una serie di priorità su cui le Università devono investire per innovare le loro pratiche didattiche. Si tratta di principi che sanciscono l'importanza della didattica come mission degli Atenei e l'importanza di porre gli studenti al centro dei processi di insegnamento-apprendimento e delle strategie di innovazione degli stessi.

Fra questi vale la pena di citarne i primi, che pongono particolare enfasi sugli studenti e sulle loro competenze.

1. L'esperienza di apprendimento dell'istruzione superiore alimenta e consente la crescita degli studenti come cittadini attivi e responsabili, pensatori critici, risolutori di problemi, attrezzati per l'apprendimento permanente.

2. L'apprendimento e l'insegnamento sono centrati sul discente. L'Università dovrebbe fornire opportunità di apprendimento su misura, che diano risposta ai bisogni e che tengano conto delle capacità di studenti diversi. Gli studen- 
ti sono attivamente coinvolti come co-creatori in tutti gli aspetti dell'esperienza formativa e condividono la responsabilità per il proprio apprendimento.

3. L'impegno per l'apprendimento e l'insegnamento è parte integrante dello scopo, della mission e della strategia dell'Università. Per migliorare l'apprendimento e l'insegnamento è necessario che la mission rifletta lo scopo e i valo-

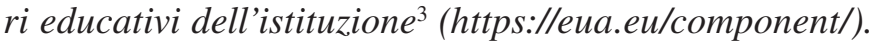

A fronte di queste indicazioni, si evidenzia l'importanza di conoscere quegli aspetti metacognitivi che - trasversali ai contenuti disciplinari e parti integranti delle competenze professionali - consentono agli studenti una padronanza dei saperi e delle abilità che vengono messe in gioco durante un percorso universitario, e che dovranno poi essere utilizzate efficacemente dai laureati quando si troveranno nel mondo del lavoro o in ulteriori percorsi di istruzione superiore. Aspetti quali il senso di autoefficacia, una motivazione intrinseca all'apprendimento, la capacità di utilizzare adeguate strategie di regolazione dell'apprendimento, di gestione degli insuccessi e degli errori e una buona fiducia nelle proprie possibilità di apprendere, sono tutte dimensioni metacognitive ritenute essenziali per una buona acquisizione di competenze, all'interno di qualsiasi profilo professionale.

La possibilità, per i docenti universitari, di disporre di valide e dettagliate informazioni su queste caratteristiche dei loro studenti rappresenta un elemento chiave per riflettere sulla propria didattica e assumere decisioni in merito al suo miglioramento.

Il dibattito internazionale su queste tematiche è oggi molto ampio e, in vari settori di ricerca connessi all'higher education, sono state messe a punto valide scale di rilevazione delle caratteristiche metacognitive degli studenti. Di seguito, vengono brevemente illustrati gli strumenti di cui si è fatto uso nei progetti pilota dell'Alma Mater Studiorum Università di Bologna.

\section{Strumenti per rilevare le caratteristiche degli studenti}

In entrambi gli studi pilota sono stati scelti come strumenti di rilevazione delle caratteristiche degli studenti in termini di strategie per l'apprendimento due questionari, somministrati longitudinalmente al primo e al terzo anno di corso.

Per il CdS in Infermieristica è stato scelto il test standardizzato Questionario sulle Strategie di Apprendimento - QSA (Pellerey, 1996) poiché considerato valido a rilevare strategie cognitive ed affettive riportate anche nel Tuning

\footnotetext{
${ }^{3}$ EUA Enhancing the education mission of European universities: A proactive response to change https://eua.eu/component/.
} 
Project for Nursing ${ }^{4}$, condiviso all'interno di due focus group che hanno visto coinvolti alcuni membri del Consiglio di CdS e alcuni infermieri che si occupano della formazione clinica degli studenti. Il QSA rileva il livello di consapevolezza e di capacità di gestione dei processi e delle strategie di apprendimento degli studenti. È un questionario di autopercezione concepito per gli studenti al termine del percorso di obbligo scolastico o formativo. Il questionario QSA si struttura attorno a quattordici fattori che rappresentano altrettante componenti della competenza strategica degli allievi in relazione all'apprendimento. Sette di questi fattori sono di natura prevalentemente cognitiva e sette di natura prevalentemente affettiva. La scelta del QSA è stata orientata dall'interesse verso le dimensioni indagate, in linea con quanto emerso nei focus group, con la consapevolezza di una certa distanza di età fra lo standard di riferimento su cui il QSA è stato validato nel 1996 (primo anno della scuola secondaria superiore) e gli studenti del primo anno di università coinvolti nella ricerca.

Nello studio pilota presso il Corso di Laurea in Economics and Finance è stato costruito un Questionario studenti ad hoc, composto da diverse scale standardizzate: la dimensione "strategie di apprendimento" è una scala composta da 18 item derivata dal QPA - Questionario sui Processi di Apprendimento - Superiori e Università di K. Poláček (2005) e misura quanto gli studenti siano capaci di utilizzare diverse modalità di approccio allo studio, consolidare conoscenze attraverso mediatori didattici (mappe, schemi, tabelle), autoregolare il proprio processo di apprendimento, gestire situazioni di ansia. Una seconda dimensione del questionario riguarda l'autoefficacia nello studio universitario e due sono le sottoscale di riferimento: "autoefficacia e autoregolazione dell'apprendimento"s, riferita alle capacità dello studente di organizzazione e pianificazione del proprio progetto di studio universitario; "autoefficacia orientata alla prestazione", con una messa a fuoco sul raggiungimento di risultati in termini di prestazione (sostenere molti esami con voti soddisfacenti).

Sono state inoltre aggiunti alcuni item estratti dal Questionario Monitoraggio Obbligo Formativo Integrato (Lodini, Luppi e Vannini, 2004) rispetto alla soddisfazione formativa relativa sia alle percezioni e valutazioni deli studenti delle modalità didattiche dei docenti universitari sia alle aspettative rispetto al CdS. Infine, è stata somministrata la Academic motivation scale (CEGEP) ${ }^{7}$

${ }^{4}$ Tuning Project. Introduzione al Tuning Educational Structures in Europa. Il contributo delle università al Processo di Bologna. Reperibile in: www.unideusto.org/tuningeu.

${ }^{5}$ Self-efficacy for self-regulated learning scale: adattata dalla Self-Efficacy for Self-Regulated Learning Scale (Zimmerman, Bandura e Mattinez-Pons, 1992)

${ }^{6}$ Subscale B: Self-efficacy for academic achievement, adattata dall'Academic Self-Efficacy Scale (Roeser, Midgley e Urdan, 1996) Items: 11-12.

${ }^{7}$ Robert J. Vallerand, Luc G. Pelletier, Marc R. Blais, Nathalie M. Brière, Caroline B. Senécal, Évelyne F. Vallières, 1992-1993, Educational and Psychological Measurement, vols. 52 and 53. 
sui fattori instriseci ed estrinseci relativi alla dimensione motivazionale negli studi universitari.

\section{Campioni di riferimento e modalità di rilevazione}

Il Questionario sulle Strategie di Apprendimento - QSA è stato somministrato a 168 studenti e studentesse iscritti al primo anno del CdS in Infermieristica nell'anno accademico 2013/2014 e, allo stesso gruppo al terzo anno, nell'anno accademico 2015/2016 (160 rispondenti sui 168 della prima rilevazione).

Poco dopo la prima rilevazione è stata organizzata una somministrazione ad altri studenti iscritti al primo anno dell'Università di Bologna (a.a 2014/15). La somministrazione del questionario è stata realizzata grazie ad un link costruito attraverso Survio, un software per questionari e sondaggi. Il link è stato diffuso attraverso pagine Facebook di gruppi chiusi di alcuni corsi di studio universitari: Economia Mercati e Istituzioni; Storia; Scienze Statistiche; Scienze Politiche; Ingegneria Gestionale; Lingue e Letterature Straniere; Lettere; Ingegneria Chimica; Dams. Hanno risposto al questionario on line 92 studenti.

Nel complesso gli studenti universitari che hanno partecipato alla prima rilevazione sono 281, in prevalenza femmine (221 studentesse contro 61 studenti), l'età media è di 20,5 anni. Per ogni link condiviso sulle bacheche pubbliche dei gruppi precedentemente elencati sono stati specificati in allegato le modalità di compilazione e gli obiettivi che si volevano raggiungere attraverso questa indagine.

Infine, il questionario è stato somministrato nell'anno scolastico 2014/2015 ad alcune classi prime di scuole secondarie superiori scelte in relazione alla possibilità di accesso diretto da parte del gruppo di ricerca.

A tal proposito sono stati contattati i dirigenti scolastici di diversi istituti superiori di alcune province di Novara. Si è rinunciato a un campionamento probabilistico delle scuole per garantire la possibilità di raccogliere dati su varie differenti indirizzi di scuola secondaria in tempi rapidi e con la piena collaborazione di dirigenti e insegnanti, condizione fondamentale per raccogliere dati validi e attendibili, dato l'impegno in termini di tempo che la compilazione del QSA richiede (circa 45 minuti).

L'analisi degli esiti nelle scale cognitive e affettive del QSA ha riguardato il confronto tra i risultati degli allievi al primo anno del Corso di Laurea in Infermieristica a Bologna, la rilevazione su matricole di diversi Corsi di Laurea dell'ateneo bolognese (2015) e gli studenti di scuola secondaria superiore della provincia di Novara coinvolti nella rilevazione del 2015. Tutti questi risultati sono stati letti in relazione allo standard su cui il QSA è stato validato: studenti del primo anno della scuola secondaria superiore e della formazione professionale (1996). 
Il Questionario studenti nel Corso di Laurea triennale in Economics and Finance è stato somministrato a tutte e tre le coorti di studenti nell'a.a. 2016-17 come "dati di ingresso" e successivamente nell'a.a. 2017-18 agli studenti del I anno di corso nel mese di dicembre 2017 e agli studenti al II e III anno nel mese di maggio 2018.

I dati dell'a.a. 2016/17 sulle coorti del I e del II anno (corrispondenti rispettivamente al II e III anno nell'a.a. 2017/18) sono stati elaborati diacronicamente al fine di rilevare cambiamenti nelle competenze metacognitive indagate. Gli studenti che hanno compilato il questionario nelle due annualità sono complessivamente 369 su un totale di circa 550 studenti iscritti al CdS.

Il Questionario è stato somministrato in diverse lezioni, a seconda degli anni di corso, con una versione on line accessibile dagli studenti non frequentanti. L'analisi dei risultati è, come per lo studio pilota nel CdS in Infermieristica, presentata nei paragrafi successivi.

\section{Presentazione dati CdS in Infermieristica e ricadute sulla progettazione didattica}

Nei Grafici 1 e 2 sono presentati i confronti fra i punteggi ottenuti dagli studenti universitari (le matricole del corso di Laurea in Infermieristica e quelle di altri corsi di studi), lo standard nazionale QSA e gli studenti del primo anno di scuola secondaria superiore coinvolti nella rilevazione dell'a.s. 2014/15. Soffermandosi sulle medie nelle scale cognitive, il diagramma a barre riassuntivo mette in evidenza una situazione in cui, per la maggior parte delle scale, il campione degli studenti universitari è assimilabile allo standard dei quindicenni della rilevazione dell'a.s. 1996/97, mentre gli studenti di scuola superiore della rilevazione odierna mostrano, nella maggior parte delle scale, livelli inferiori. Nello specifico fanno riflettere i punteggi sulle scale dell'autoregolazione, l'uso di organizzatori semantici, l'autointerrogazione in cui i punteggi degli attuali universitari sono analoghi a quelli dei quindicenni del 1996/97. Nelle scale relative alla difficoltà di concentrazione, alla disponibilità alla collaborazione e al disorientamento degli studenti universitari hanno, addirittura, punteggi più bassi dello standard. Solo sulle strategie elaborative i punteggi medi degli studenti universitari sono più alti di quelli degli studenti di scuola superiore. Se ci si sofferma sui dati dei quindicenni della rilevazione odierna emergono, in particolare, punteggi superiori nelle scale relative alla difficoltà di concentrazione e al disorientamento. 
Graf. 1 - Punteggi medi nelle scale cognitive QSA: grafico riassuntivo rilevazioni università (studio Infermieristica a.a. 2013/2014 + studio a.a. 2014/2015), scuole secondarie a.s. 2014/2015 e standard nazionale a.s. $1996 / 1997$

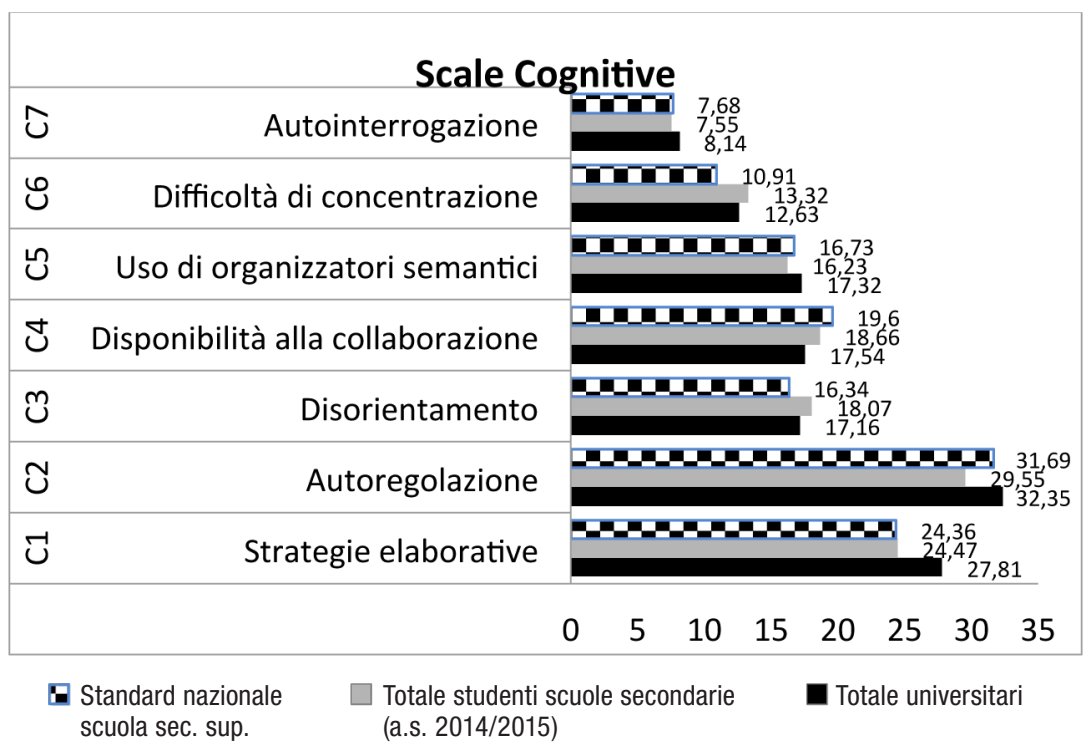

Nota: Le scale C3 e C6 risultano capovolte: i punteggi vanno letti come «positivi» se sono bassi, e «negativi» 0 «preoccupanti» se sono alti.

Il Grafico 2 ci permette di comparare i punteggi medi nelle scale affettive fra universitari, studenti delle scuole secondarie della rilevazione 2014/15 e lo standard del 1996/97. Anche in questo caso gli studenti universitari raggiungono punteggi analoghi allo standard dei quindicenni in varie scale: l'ansietà di base, la mancanza di perseveranza, l'attribuzione a cause controllabili $\mathrm{e}$ il controllo delle interferenze emotive. I loro punteggi sono lievemente peggiori rispetto alla volizione, all'attribuzione a cause incontrollabili e alla percezione di competenza. Gli studenti delle scuole secondarie della rilevazione 2015/16 ottengono punteggi decisamente peggiori rispetto allo standard nelle scale relative alla volizione e alla mancanza di perseveranza (i punteggi sono più alti poiché la scala è capovolta) e medie un po' più basse nell'attribuzione a cause controllabili e nella percezione di competenza. 
Graf. 2. - Punteggi medi nelle scale affettive QSA: grafico riassuntivo rilevazioni università (studio Infermieristica a.a. 2013/2014 + studio a.a. 2014/2015), scuole secondarie a.s. 2014/2015 e standard nazionale a.s. $1996 / 1997$

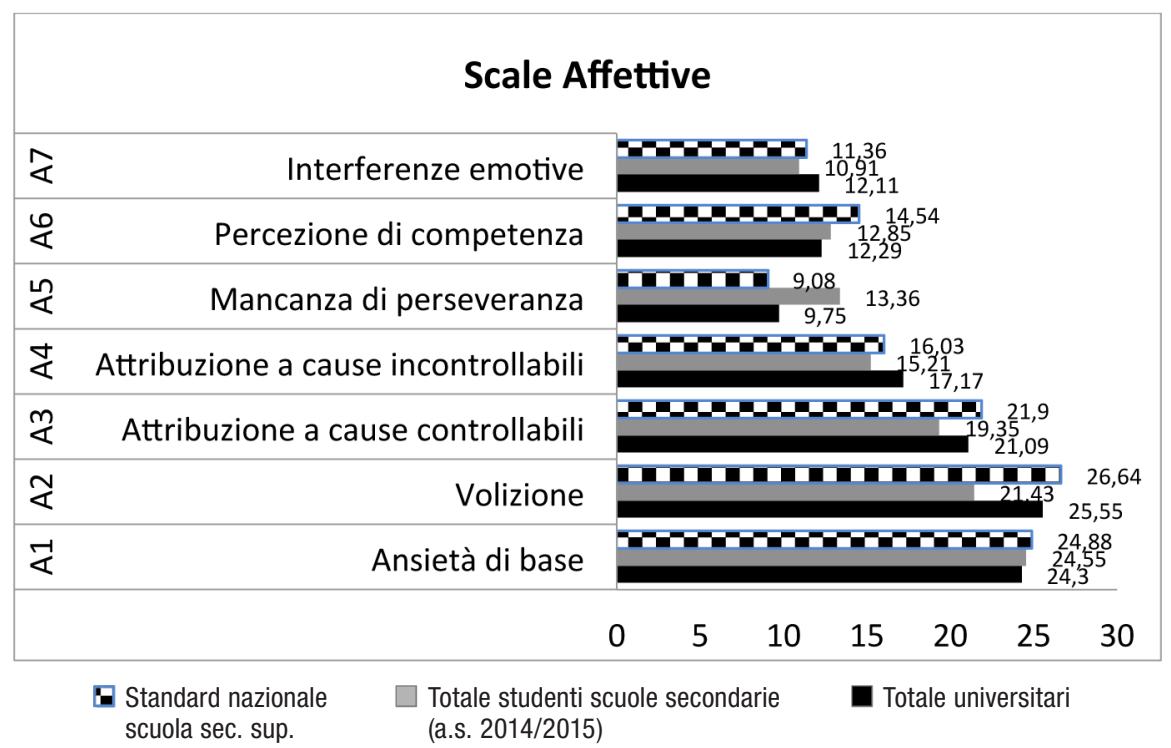

Nota: Le scale A1, A5, A7 risultano capovolte: i punteggi vanno letti come «positivi» se sono bassi, e «negativi» 0 «preoccupanti» se sono alti.

I dati nel loro complesso fanno emergere alcune differenze, ma anche molti punti di vicinanza, nonostante le differenze di età. Se, da un lato, il campione degli studenti universitari mostra di possedere più strategie elaborative e maggior autoregolazione rispetto allo standard e alle scuole secondarie, dall'altro, mette in evidenzia un maggiore disorientamento e una minor disponibilità alla collaborazione. Gli universitari riportano maggiori difficoltà di concentrazione rispetto allo standard, ma una buona capacità di auto interrogazione e di uso di organizzatori semantici. Nell'ambito delle scale affettive gli studenti universitari hanno ottenuto punteggi molto simili allo standard e alle scuole secondarie nell'ansietà di base e attribuzioni a cause controllabili, mentre punteggi superiori allo standard rispetto all'attribuzione a cause incontrollabili, alla mancanza di perseveranza e alle interferenze emotive. Inoltre, gli studenti universitari hanno mostrato punteggi inferiori nella volizione solo rispetto allo standard e nella percezione di competenza rispetto a standard e scuole secondarie.

Nella lettura complessiva dei dati emerge un quadro in cui gli studenti universitari non si distaccano in modo così netto dallo standard, salvo su alcune singole scale. D’altro canto, il gruppo di studenti quindicenni coinvolto nella re- 
cente somministrazione mostra, nel complesso, punteggi peggiori rispetto ai coetanei di venti anni fa, seppure con qualche lieve differenza a seconda dell'indirizzo scolastico.

La rilevazione effettuata al termine del Corso di Studi in Infermieristica, i cui dati di dettaglio non vengono qui riportati per ragioni di spazio, ha mostrato un miglioramento nei punteggi degli studenti, sebbene ancora non si possa parlare di uno scarto importante rispetto allo standard dei quindicenni (Luppi, Benini, 2017).

\section{Alcune considerazioni sulle competenze metacognitive degli studenti universitari}

I dati degli studenti del primo anno di più corsi di Laurea dell'Università degli Studi di Bologna, confrontati con gli studenti delle prime classi di scuola secondaria superiore (lo standard del 1996 e il campione di studenti del 2015) mettono in evidenza una "regressione" delle strategie di apprendimento da un punto di vista cognitivo e affettivo. I dati nel post-test mostrano un quadro di miglioramento e di incremento nell'acquisizione di queste competenze, ma in modo non così marcato né omogeneo, in sostanza gli studenti di Infermieristica che si avvicinano alla laurea mostrano ancora una serie di fragilità sulle strategie metacognitive e punteggi medi non sempre distanti dallo standard come ci si aspetterebbe.

Si tratta di un risultato non generalizzabile all'intera popolazione studentesca universitaria e di scuola secondaria superiore. L'indagine, infatti, è stata condotta su un campione degli universitari non probabilistico e fortemente disomogeneo; ugualmente non probabilistico e alquanto ridotto rispetto allo standard del 1996 è il campione di studenti della scuola secondaria di secondo grado.

Tuttavia, già da questi primi dati, è possibile avviare alcune riflessioni su quanto è emerso dall'analisi dei QSA distribuiti. Se è vero che le competenze metacognitive per l'apprendimento sono diminuite nell'arco temporale di vent'anni, sorgono spontanee alcune domande formulate per comprendere e chiarire l'entità di tale fenomeno. E dunque: come le agenzie intenzionalmente educative valorizzano e trasferiscono formalmente le strategie per l'apprendimento agli studenti? In passato i processi metacognitivi venivano sostenuti e veicolati in modo più marcato e con strumenti diversificati rispetto ad oggi? Come la scuola, l'università e gli altri setting formativi supportano concretamente lo sviluppo di tali competenze a fronte anche all'enfasi che si sta dando alla promozione delle Life Skills?

Occorrerebbe, pertanto, avviare una riflessione mirata per comprendere come la didattica, in modo particolare, promuova e potenzi le competenze me- 
tacognitive per l'apprendimento negli studenti sia della scuola secondaria, sia all'università.

Anche a partire da questi dati il Corso di Laurea in Infermieristica e l'Università di Bologna nel suo complesso ha avviato una riflessione mirata per comprendere come la didattica, in modo particolare, possa promuovere e potenziare le competenze metacognitive per l'apprendimento negli studenti.

\section{Presentazione dati del Corso di Laurea in Economics and Finance (CLEF) e im- plicazioni per la didattica}

Essendo il primo studio pilota ancora in corso di svolgimento, è opportuno sottolineare che i risultati che vengono di seguito presentati risultano ancora parziali ${ }^{8}$. Si ritiene comunque interessante presentare alcune dimensioni emerse dall'analisi dei dati, sia per quanto riguarda la direzione di ricerca obiettivirisultati sia per quanto concerne processi e contesti. Si tratta di mere esemplificazioni - che prendono in esame solo alcuni dati inerenti le caratteristiche degli studenti e le autovalutazioni dei docenti sulla didattica - utili a comprendere come si sia proceduto, nel gruppo di ricerca, per l'analisi dei dati e l'individuazione di aspetti importanti su cui concentrare l'attenzione nelle fasi di ri-progettazione del CLEF e dei suoi processi didattici.

\section{Strategie di apprendimento}

La rating scale di ogni item è articolata su valori da 1 (quasi mai) a 5 (quasi sempre), per un punteggio massimo pari a 90. La Tabella 1 illustra sinteticamente la media e la deviazione standard della dimensione relativamente ai risultati degli studenti del CLEF. Per questa scala è possibile fare riferimento ai punteggi standardizzati del QPA-D (media e deviazione standard STD) definiti su un campione normativo di studenti universitari provenienti da diversi corsi di studi. Un'analisi in profondità del costrutto dei singoli item ha permesso, in sede di confronto con i docenti del CLEF, una riflessione sugli stili di apprendimento dei loro studenti nei differenti anni di corso, sulle loro modalità di approccio allo studio e sull'uso di strategie focalizzate su un apprendimento mnemonico oppure sull'uso di strategie - più desiderabili - di apprendimento significativo dei contenuti disciplinari.

${ }^{8}$ Sulla base di accordi con l'Ateneo di Bologna, si presentano solo alcuni dati anche in ragione di aspetti di privacy e di cautela rispetto al lavoro di riflessione e riprogettazione in atto nel gruppo di ricerca. 
La seguente tabella illustra sinteticamente la media e la deviazione standard della dimensione e il riferimento ai punteggi standardizzati del QPA-D.

Tab. 1 - Elaborazione dati questionario studenti relativi alla dimensione Strategie di apprendimento

\begin{tabular}{|c|c|c|c|c|c|}
\hline & $\begin{array}{l}\text { Media } \\
\text { CLEF }\end{array}$ & $\begin{array}{l}\text { dev standard } \\
\text { CLEF }\end{array}$ & $\begin{array}{l}\text { Indice di variazione } \\
\text { in } \%\end{array}$ & $\begin{array}{l}\text { Media } \\
\text { STD }\end{array}$ & Dev STD \\
\hline \multicolumn{4}{|c|}{ a.a. $2016 / 2017$} & \multirow{8}{*}{67,45} & \multirow{8}{*}{9,52} \\
\hline I anno & 61,61 & 11,18 & 18,15 & & \\
\hline II anno & 59,20 & 9,93 & 16,77 & & \\
\hline III anno & 57,48 & 9,21 & 16,02 & & \\
\hline \multicolumn{4}{|c|}{ a.a. $2017 / 2018$} & & \\
\hline I anno & 61,71 & 11,89 & $19 \%$ & & \\
\hline II anno & 60,41 & 11,44 & $19 \%$ & & \\
\hline III anno & 61,20 & 8,67 & $14 \%$ & & \\
\hline
\end{tabular}

La media dei punteggi relativi alle strategie di studio si colloca, per tutte le coorti di studenti, al di sotto della media riferita al campione normativo. Si è deciso pertanto di approfondire i dati seguendo le indicazioni del QPA, individuando profili di soggetti universitari in apprendimento.

Un approfondimento sulla dimensione Strategie di apprendimento è stato condotto attraverso la trasformazione dei punteggi grezzi in stanine $(\mathrm{N}=295)$. Nel volume di riferimento (Poláček, 2005) sono esplicitate le tabelle di conversione; si riportano in Tabella 2 le frequenze (n.studenti) per livelli di stanine; è stato evidenziato in azzurro il livello con la maggior frequenza in termini di n. di studenti. I differenti livelli di stanine corrispondono, secondo il quadro teorico di riferimento esplicitato da Poláček, a profili di soggetti universitari in apprendimento; ogni profilo evidenzia l'efficacia o i punti di criticità relativi alle strategie da mettere in atto come processo di autoregolazione del proprio modo di apprendere, organizzare contenuti e monitorarne l'acquisizione. 
Tab. 2 - Frequenze (valori assoluti) di studenti CLEF per livelli di stanine della dimensione Strategie di apprendimento

\begin{tabular}{|l|c|c|c|c|}
\hline & $\begin{array}{c}\text { Studenti } \\
\text { I anno } \\
\mathbf{2 0 1 6 - 2 0 1 7}\end{array}$ & $\begin{array}{c}\text { Studenti } \\
\text { II anno } \\
\mathbf{2 0 1 6 - 2 0 1 7}\end{array}$ & $\begin{array}{c}\text { Studenti } \\
\text { III anno } \\
\mathbf{2 0 1 6 - 2 0 1 7}\end{array}$ & $\begin{array}{c}\text { Studenti } \\
\text { I anno } \\
\mathbf{2 0 1 7 - 2 0 1 8}\end{array}$ \\
\hline Livello 1 & 10 & 8 & 6 & 12 \\
\hline Livello 2-3 & 20 & 12 & 7 & 20 \\
\hline Livello 4-5-6 & 36 & 19 & 9 & 33 \\
\hline Livello 7-8 & 5 & 2 & 1 & 6 \\
\hline Livello 9 & 1 & - & - & 2 \\
\hline Totale studenti & 72 & 41 & 23 & 73 \\
\hline
\end{tabular}

\section{Autoefficacia, autoregolazione dell'apprendimento e orientamento alla prestazione}

La rating scale della dimensione "autoefficacia e autoregolazione dell'apprendimento", strettamente legata alle strategie di apprendimento poiché caratterizzante componenti metacognitive delle competenze degli studenti, ha punteggi da 1 (molto debole) a 5 (molto forte). Per tutti gli anni del CLEF la media dei risultati, presentata in Tabella 3, si colloca in una fascia medio-alta (punteggio medio 3,7 per l'a.a. 2016/2017 e 3,5 per l'a.a. 2017/2018). Interessante osservare che la coorte di studenti al primo anno nell'a.a. 2016/2017 presenta per l'a.a. 2017/2018 una media più bassa di autoefficacia nell'autoregolazione del proprio apprendimento, con un'alta percentuale di variazione all'interno del gruppo di studenti (indice di variazione del 56\%).

Tab. 3 - Elaborazione dati questionario studenti relativi alla dimensione Autoefficacia e autoregolazione dell'apprendimento

\begin{tabular}{|c|c|c|c|}
\hline \multicolumn{4}{|c|}{ A.a. 2016/17 } \\
\hline & Media CLEF & dev standard CLEF & Indice di variazione \\
\hline I & 3,8 & 0,5 & $13 \%$ \\
\hline$I I I$ & 3,8 & 0,4 & $11 \%$ \\
\hline \multicolumn{5}{|c|}{ A.a. 2017/18 } \\
\hline I & 3,6 & 0,5 & $14 \%$ \\
\hline II & 3,6 & 0,7 & $19 \%$ \\
\hline III & 3,0 & 1,7 & $56 \%$ \\
\hline
\end{tabular}


Analogamente alla subscale precedente, sebbene i risultati della scala " $a u$ toefficacia orientata alla prestazione" siano mediamente in una fascia elevata (media del 3,9/5 nell'a.a. 2016/2017 e media del 3,7/5 nell'a.a. 2017/2018), la coorte degli studenti del II anno (a.a. 2017/18) presenta una media più bassa dell'anno accademico precedente, con un alto indice di variazione (57\%). Come si evince dai dati presentati nella Tabella 4, gli studenti del CLEF hanno un valore medio alto in tutti e tre gli anni di corso sia nell'autoefficacia e autoregolazione dell'apprendimento (solo lievemente più bassa), sia nell'autoefficacia orientata alla prestazione. L'omogeneità dei gruppi (si veda l'indice di variazione percentuale) è abbastanza elevata, soprattutto per quanto concerne il senso di autoefficacia di tipo più intrinseco, ossia orientato alla propria capacità di autoregolare l'apprendimento. Più disomogenea la distribuzione dell'efficacia orientata alla prestazione (dunque quella di tipo più estrinseco rispetto all'obiettivo dell'apprendimento), in particolare tra gli studenti del III anno.

Tab. 4 - Elaborazione dati questionario studenti relativi alla dimensione Autoefficacia orientata alla prestazione

\begin{tabular}{|c|c|c|c|}
\hline \multicolumn{4}{|c|}{ A.a. 2016/17 } \\
\hline & Media CLEF & dev standard CLEF & Indice di variazione \\
\hline I & 4,0 & 0,8 & $20 \%$ \\
\hline II & 4,0 & 0,7 & $18 \%$ \\
\hline III & 3,9 & 0,9 & $23 \%$ \\
\hline \multicolumn{4}{|c|}{ A.a. 2017/18 } \\
\hline I & 4,0 & 0,8 & $20 \%$ \\
\hline II & 3,0 & 1,7 & $57 \%$ \\
\hline III & 4,0 & 0,7 & $18 \%$ \\
\hline
\end{tabular}

\section{Alcune analisi inferenziali}

Al fine di individuare i principali ambiti di riprogettazione e miglioramento del CdS, sono state compiute inoltre analisi di tipo inferenziale sull'insieme delle coorti analizzate nei due anni di studio pilota.

Come si può osservare dai principali modelli di regressione che sono stati definiti, l'influenza di alcuni ambiti metacognitivi sui risultati degli studenti (in termini di votazione agli esami e di numero di CFU acquisiti) è molto rilevante. Vengono infatti presentati di seguito solo alcuni dei modelli testati attraverso modelli di moderazione o mediazione usando le macro PROCESS per SPSS (Hayes, 2013). 
Nello specifico, le analisi evidenziano come sui risultati degli studenti del I anno di corso siano soprattutto la motivazione allo studio e il senso di autoefficacia gli aspetti più influenti; va tuttavia notata (si veda Figura 2) un'importante influenza di una variabile moderatrice: il possesso di buone strategie di regolazione dell'apprendimento tendono ad interagire soprattutto la motivazione di tipo intrinseco allo studio e, di conseguenza, la sua azione positiva sui risultati finali agli esami. Il grafico (Figura 2) mostra infatti con chiarezza come a un alto livello di motivazione intrinseca sia la presenza di un livello alto di strategie di apprendimento ad associarsi ad una più elevata media dei voti.

Fig. 2 - Esiti analisi sulle caratteristiche degli studenti al I anno di corso
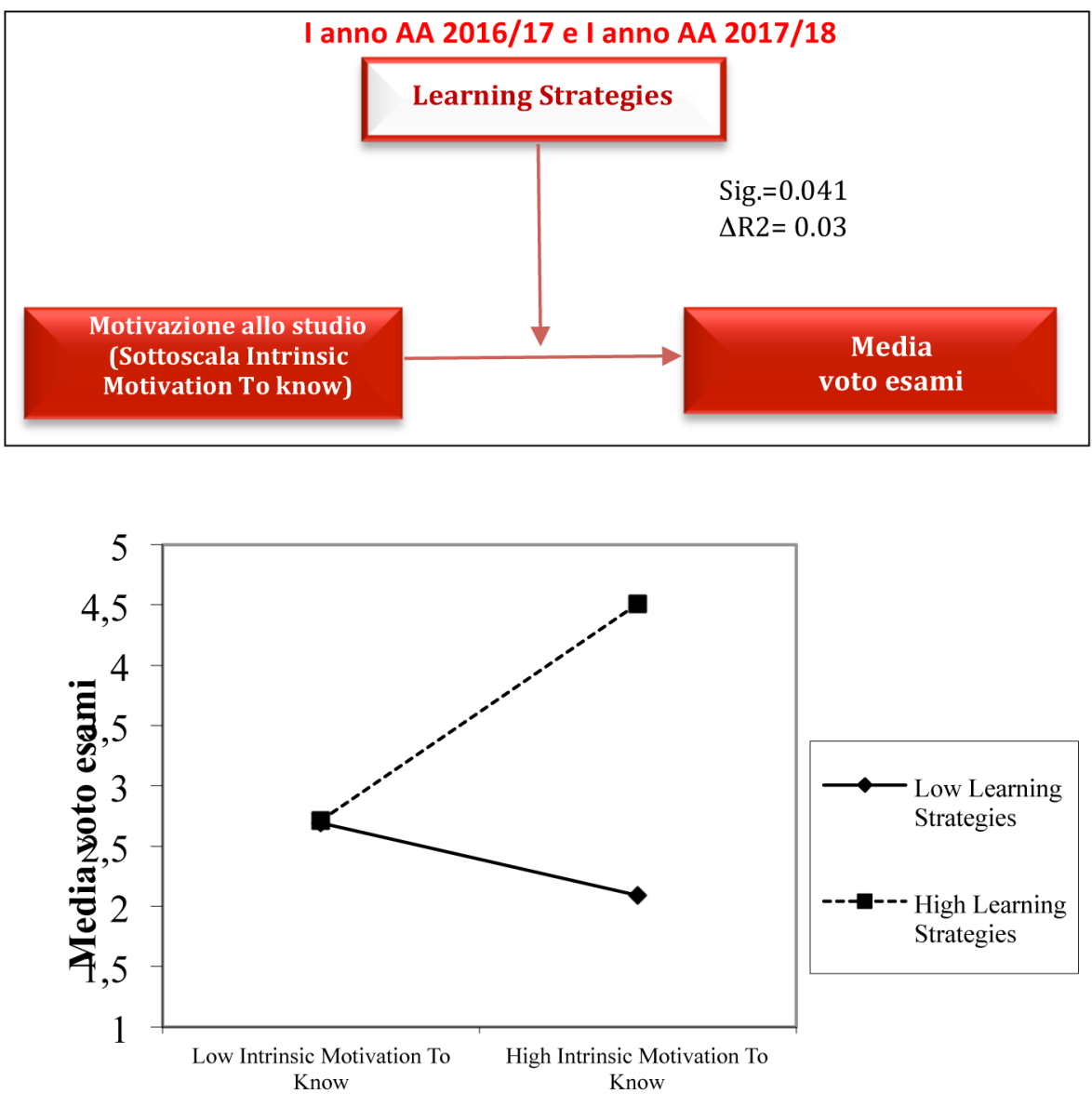
Fig. 2 - Segue

\begin{tabular}{|l|l|l|}
\hline & Coeff. & $p$ \\
\hline Media Learning Strategies & 0.61 & 0,126 \\
\hline Intrinsic Motivation To Know & 0.30 & 0.237 \\
\hline Interazione Mot. e LS & 0.60 & 0.041 \\
\hline
\end{tabular}

In merito alle coorti rilevate del II anno di corso, la variabile "possesso di adeguate strategie di apprendimento" (si veda Figura 3) emerge come dimensione che innesca il processo che porta al raggiungimento di positivi risultati finali. I risultati della mediazione in serie riportati in Figura 3 mostrano che essa influenza il senso di autoefficacia e, allo stesso tempo, promuove un adeguato orientamento al futuro, coerentemente col profilo del CdS. Occorre qui sottolineare che l'effetto di buone strategie di apprendimento è rilevante sia in modo diretto sui risultati agli esami, ma anche attraverso l'effetto indiretto (si veda Figura 4) dell'autoefficacia. Anche per quanto riguarda l'orientamento al futuro, indicatore importante anche in termini di carriera futura si può osservare sia l'effetto diretto delle strategie di apprendimento sia quello indiretto della percezione di autoefficacia e della media dei voti.

Fig. 3 - Esiti analisi sulle caratteristiche degli studenti al II anno di corso

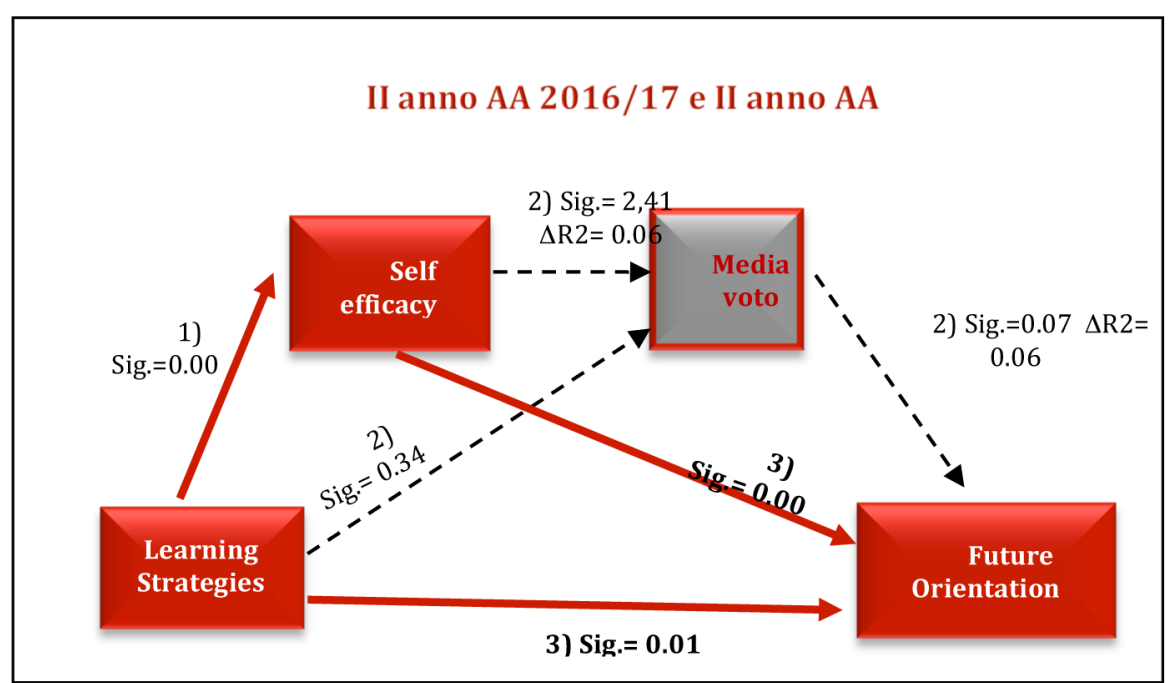


Fig. 4 - Esiti analisi sulle caratteristiche degli studenti al II anno di corso

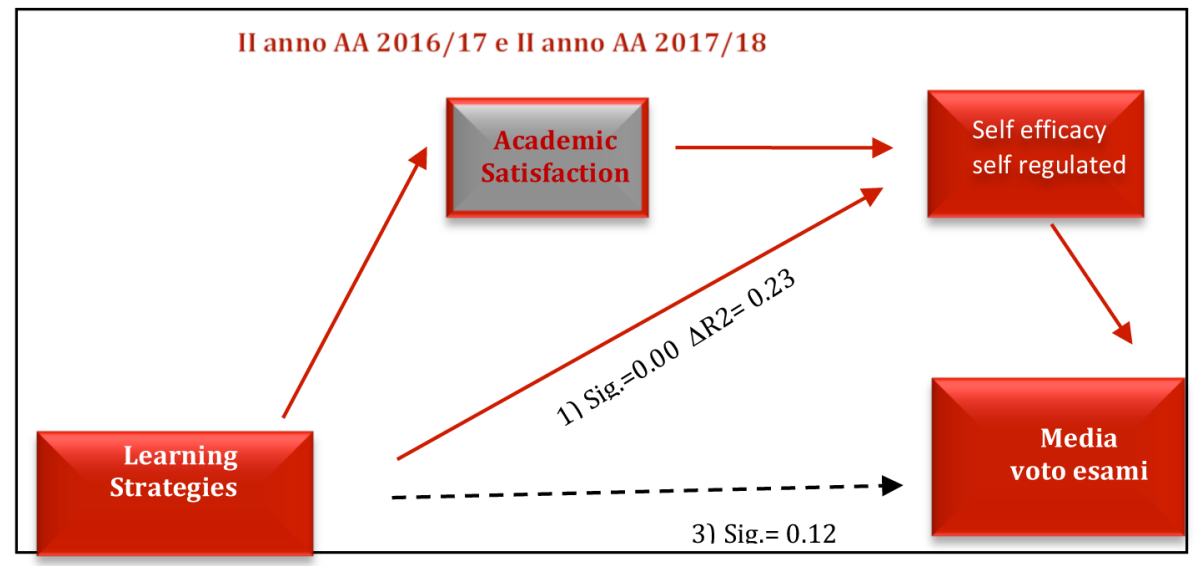

La soddisfazione (valutazione/percezione positiva degli studenti delle modalità didattiche dei docenti) per la didattica universitaria assume infine un ruolo davvero determinante nelle coorti degli studenti del III anno.

In questo caso, osserviamo (si veda Figura 5 che mostra la sintesi delle analisi di regressione) che le due variabili che maggiormente incidono sui risultati finali (in termini di numero di esami e di CFU acquisiti durate l'ultimo anno di $\mathrm{CdS}$ ) sono nuovamente la motivazione intrinseca allo studio (Beta e, con un impatto davvero importante, la valutazione positiva della didattica in aula dei docenti (Beta $=0,90)$, in particolare per quanto concerne valutazioni positive sulla chiarezza delle spiegazioni, l'uso di metodologie didattiche attente alle esigenze degli studenti, ecc.

I risultati presentati in questo lavoro rappresentano solo una parte di quelli disponibili, ma aiutano a capire la logica con cui sono stati analizzati i bisogni e con cui possono essere impostate azioni di orientamento, accompagnamento e supporto.

Dall'insieme dei dati è emerso che le principali aree di intervento su cui i colleghi docenti hanno deciso di lavorare per migliorare la qualità del loro $\mathrm{CdS}$ e della loro didattica universitaria sono le seguenti:

- Orientamento in ingresso degli studenti, in particolare nella direzione di una consapevolezza delle abilità necessarie per affrontare il $\mathrm{CdS}$ e nella definizione del profilo professionale.

- Motivazione allo studio, ovvero riflettere e lavorare sull'attributional retraining per modificare le percezioni degli studenti ed aiutarli ad attribuire la scarsa performance a cause modificabili (es. impegno e strategie di apprendimento efficaci) piuttosto che a cause immutabili (es. abilità accademica come dote naturale ed intelligenza). 
Fig. 5 - Esiti analisi sulle caratteristiche degli studenti al III anno di corso

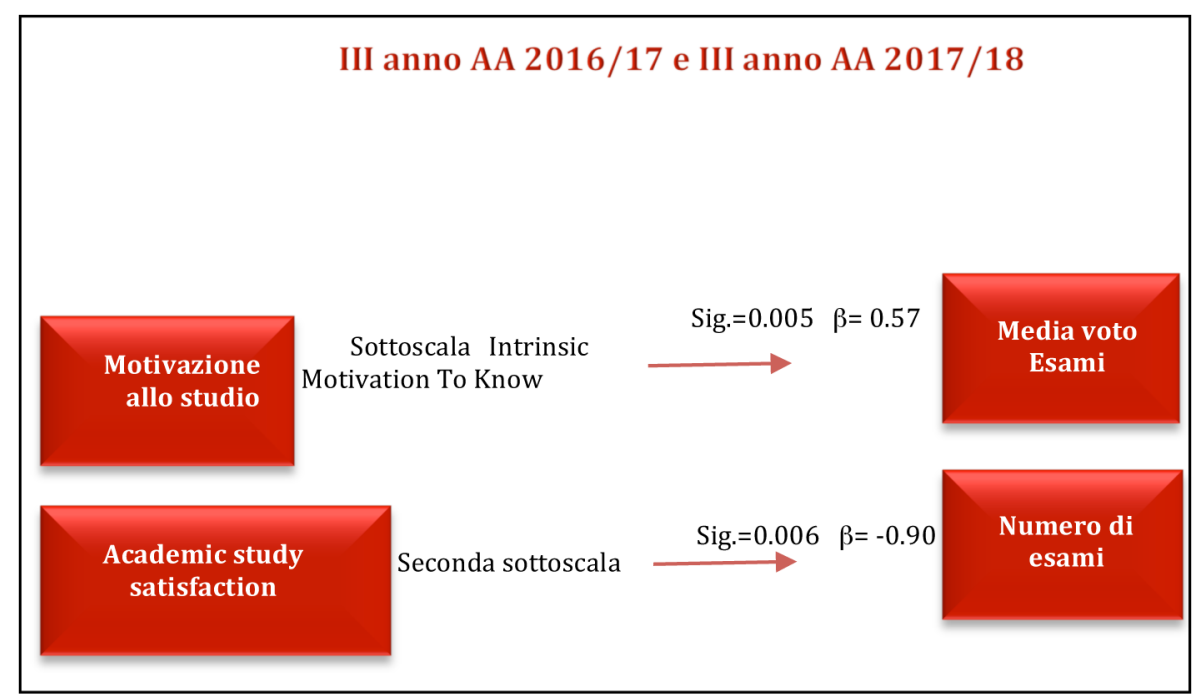

- Strategie di apprendimento: formare gli studenti al metodo di studio e utilizzare in aula strategie didattiche che favoriscano autovalutazione e controllo delle proprie strategie di apprendimento. Ne sono esempi l'utilizzo in aula del formative assessment e del feedback formativo per aumentare le capacità di autovalutazione degli studenti e le loro strategie di controllo sull'apprendimento nei contesti di higher education.

- Orientamento in uscita: curare particolarmente l'orientamento al futuro degli studenti, affinché siano consapevoli delle reali opportunità di studio e lavoro a livello nazionale e internazionale.

Le analisi inferenziali sulle coorti di studenti e le analisi relative ai dati sui docenti hanno inoltre evidenziato come variabile-chiave su cui agire in modo determinante la soddisfazione degli studenti per i processi didattici in aula $\mathrm{e}$, specularmente, le abilità didattiche del docente in aula. Nello specifico, i docenti evidenziano il bisogno di padroneggiare strategie didattiche per favorire la metacognizione degli studenti (uso di mediatori didattici differenziati, uso del feedback formativo, uso delle nuove tecnologie); conoscere in modo diagnostico le caratteristiche metacognitive degli studenti in ingresso. 


\section{Conclusioni e sviluppi progettuali del modello}

Le prospettive teorico-metodologico sopra brevemente descritte hanno aperto un orientamento innovativo per lo sviluppo di una strategia volta a qualificare la didattica all'interno dell'Ateneo bolognese. Gli elementi chiave di questo modello possono essere sintetizzati nei seguenti punti-chiave, che rappresentano altrettante direzioni strategiche per il progetto di Ateneo:

a) la necessità di costruire un progetto di sviluppo della qualità della didattica che metta al centro i docenti e universitari, la loro possibilità di analizzare i contesti di partenza, i loro bisogni specifici (individuali e collettivi) e la loro possibilità di decidere e agire per la propria formazione;

b) la valutazione dei contesti e dei processi come base di partenza per qualsiasi percorso di miglioramento dei contesti e come esperienza concreta, per i docenti, di apprendimento e crescita professionale;

c) la messa in campo di percorsi formativi come risposta ai bisogni individuali e collettivi; percorsi che devono essere frutto di analisi e progettazioni condivise da coloro che hanno sperimentato un precedente momento di valutazione e auto-valutazione, di analisi e riflessività critica sull'esistente;

d) la convinzione che lo sviluppo di una buona competenza nella didattica universitaria non è mai disgiunta da una pratica effettiva della collegialità fra docenti e ricercatori, intesa come comunità professionale di riferimento che analizza e discute le criticità dei contesti e dei processi di insegnamento-apprendimento e ne individua - collettivamente - le strategie e le condizioni istituzionali per il miglioramento;

e) la consapevolezza che qualsiasi percorso di sviluppo della professionalità richiede accompagnamento e supporto da parte di figure di sistema competenti negli ambiti della progettazione e valutazione didattica, della psicologia delle organizzazioni, della formazione degli adulti e della conduzione di gruppi di lavoro.

Le azioni strategiche che l'Università di Bologna ha realizzato in campo si articolano su due livelli di intervento.

\section{Le azioni di livello A: Attività di Ricerca Formazione (sul modello della Formative Educational Evaluation)}

Le due esperienze pilota di ricerca valutativa e Ricerca-Formazione costituiscono la base per l'allargamento del progetto all'interno dell'Università di Bologna; esse rappresentano un prototipo per la realizzazione di ulteriori attività di formative educational evaluation su più larga scala. In particolare, nell'arco di un triennio e per passaggi successivi, vengono realizzate ulteriori esperienze di formative educational evaluation collegate a specifici Corsi di studi che si auto-candidino per partecipare al progetto. Al momento sono in fase di avvio due 
ulteriori ricerche sul Corso di Laurea in Veterinaria e sul Corso di Laurea in Ingegneria chimica e di processo.

All'interno di questi contesti vengono portate avanti azioni di raccolta sistemica di dati, analisi e confronto collettivo con i Corsi di studio, individuazione dei bisogni formativi dei docenti e messa in campo delle conseguenti azioni formative utili alla riprogettazione futura, anche sperimentando le tecniche della videoanalisi per supportare i docenti nella riflessione e riprogettazione della didattica.

Come verrà illustrato in seguito gli esiti di queste esperienze all'interno dei corsi di laurea vengono utilizzati per qualificare anche gli altri interventi di natura più trasversale al fine di arricchire l'offerta formativa e le altre esperienze rivolte ai docenti.

2. Le azioni di livello B: Percorsi specifici di formazione alla didattica attraverso metodologie innovative

Una serie di azioni trasversali, connesse alla qualificazione della didattica universitaria orientate a sensibilizzare - in modo allargato - tutto il corpo docente ai temi della qualità della didattica.

Inoltre, la diffusione, tra gli studenti Unibo, di un'offerta ampia di formazione su competenze trasversali rappresenta un'ulteriore possibilità di arricchimento del dibattito sulla didattica universitaria all'interno dell'Ateneo; la scelta e la progettazione integrata di questi specifici corsi, all'interno dei vari Consigli di $\mathrm{CdS}$, costituisce un'opportunità importante per analizzare i bisogni dei propri studenti, anche e soprattutto in rapporto alla società in cui dovranno agire esercitando competenze di cittadinanza.

I due livelli di azione sono tra loro complementari e interrelati, alimentandosi reciprocamente e rendendo effettiva una logica di innovazione basata sulla ricerca, promuovendo in modo fattivo pratiche di riflessività nella professionalità docente e creando occasioni di apprendimento esperienziale in contesti di collegialità. I primi esiti di questi percorsi hanno messo in luce risultati positivi, sia in termini di impatto sulla didattica, sia in termini di gradimento e partecipazione degli attori coinvolti. Il progetto è tutt'ora in corso di implementazione e, solo fra qualche tempo, sarà possibile offrire un quadro ampio e completo dei risultati nel loro complesso. 


\section{Riferimenti bibliografici}

Amundsen G.Y., Haakstad J. (2017). Teaching in Higher Education: consistency and change in context and role. 12th European Quality Assurance Forum, Nov. 2017. Testo disponibile al sito: www.eua.be/Libraries/EQAF-2017/p3_anders-en--haakstad.pdf?sfvrsn=0.

Becchi E., Bondioli A. (1994). La valutazione. Una pratica in via di definizione. In: Ferrari M., a cura di, La valutazione di contesti prescolari. Bergamo: Junior.

Betti M., Davila D., Martínez A., Vannini I. (2015). Una ruta hacia un sistema de aseguramiento de la calidad en Educación Superior: la experiencia del proyecto TRALL (Un percorso verso un sistema di assicurazione della qualita per L'istruzione Superiore: L'esperienza del progetto TRALL). Journal Of Educational, Cultural And Psychological Studies, 12: 77-115. DOI: 10.7358/ecps-2015-012-bett

Betti M., Vannini I. (2013). Valutare la qualità dei corsi di lifelong learning in America Latina. Alcune riflessioni teoriche e metodologiche sul disegno valutativo utilizzato nel progetto AlfaIII Trall. Ricerche di Pedagogia e Didattica, 8, 2: 45-61. DOI: 10.6092/issn.1970-2221/4036

Cherryholmes C.C. (1992). Notes on pragmatism and scientific realism. Educational Researcher, 21: 13-17. DOI: 10.3102/0013189X021006013

European Commission (2011). Reference points for the design and delivery of degree programmes in nursing. Testo disponibile al sito: www.unideusto.org/tuningeu/.

European Students' Union (ESU), 2015, Overview on student- centred learning in higher education in Europe (Brussels, ESU). Testo disponibile al sito: www.esuonline.org/wp-content/uploads/2016/07/Overview-on-Student-Centred-Learning-inHigher-Educa- tion-in-Europe.pdf.

Gaebel M., Zhang T. (2018). Trends 2018: Learning and teaching in the European Higher Education Area. European University Association.

Greene J.C. (2008). Is Mixed Methods Social Inquiry a Distinctive Methodology? Journal of Mixed Methods Research, 2(1): 7-22. DOI: 10.1177/1558689807309969

Guasti L. (2013). Competenze e valutazione metodologica. Trento: Erickson.

Hackett G., Betz N.E. (1992). Self-efficacy perceptions and the career-related choices of college students. In: Schunk D.H., Meece J.L., a cura di, Student perceptions in the classroom. Hillsdale, NJ: Erlbaum.

Harvey L., Green D. (1993). Defining Quality. Assessment and Evaluation in Higher Education, 18(1): 9-34. DOI: 10.1080/0260293930180102

Harvey L., Williams J. (2010). Fifteen years of Quality in Higher Education. Quality in Higher Education, 16(1): 3-36. DOI: 10.1080/13538321003679457

Hayes A.F. (2013). An introduction to mediation, moderation, and conditional process analysis: A regression-based approach. New York: Guilford Press.

House E.R., Howe K.R. (2003). Deliberative Democratic Evaluation. In: Kellaghan T., Stufflebeam D.L., a cura di, International Handbook of Educational Evaluation. Dordrecht-Boston-London: Kluwer.

Husén T., Postlethwaite T.N., a cura di (1994). International encyclopedia of education (2nd ed.). New York: Pergamon Press.

Kellaghan T., Stufflebeam D.L., a cura di (2003). International handbook of educational evaluation. Dordrecht - Boston - London: Kluwer. 
Le Boterf G. (1990). De la compétence: Essai sur un attracteur étrange. Paris: Les Éditions d'organisation.

Lodini E., Luppi E., Vannini I. (2004). La valutazione della qualità nell'obbligo formativo. Un'indagine esplorativa con allievi e formatori dei CFP della provincia di Bologna. Bologna: Provincia di Bologna.

Luppi E., Benini S. (2017). Valutare le strategie di apprendimento negli studenti universitari: primi risultati di una ricerca valutativa condotta all'Università di Bologna. Journal of Educational, Cultural And Psychological Studies, 16: 99-127. DOI: 10.7358/ecps-2017-016-lupp

Mertens D.M. (1998). Research Methods in Education and Psychology. Integrating Diversity with Quantitative and Qualitative Approaches. Thousand Oaks, CA: Sage Publications.

Mertens D.M. (2018). Mixed Methods Design in evaluation. Thousand Oaks, CA: Sage Publications.

Murphy J.P. (1990). Pragmatism: from Peirce to Davidson. Boulder, CO: Westview Press.

Pellerey M. (1983). Progettazione formativa: teoria e metodologia. Roma: Quaderni di formazione Isfol.

Pellerey M., Orio F. (1996). Questionario sulle strategie d'apprendimento (QSA). Roma: LAS.

Pellerey M. (2004). Competenze individuali e portfolio. Firenze Scandicci: La Nuova Italia.

Poláček K. (2005). QPA - Questionario sui Processi di Apprendimento. Firenze: O.S. Organizzazioni Speciali.

Roeser R.W., Midgley C., Urdan T.C. (1996). Perceptions of the school psychological environment and early adolescents' psychological and behavioral functioning in school: The mediating role of goals and belonging. Journal of Educational Psychology, 88(3): 408-422. DOI: 10.1037//0022-0663.88.3.408

Ryan K.E., Bradley Cousins J., a cura di (2009). The Sage international handbook of educational evaluation. Los Angeles: SAGE.

Serbati A., Zaggia C. (2012). Allineare le metodologie di insegnamento, apprendimento e valutazione ai learning outcomes. Una proposta per i corsi di studio universitari. Giornale Italiano della Ricerca Educativa, 5: 11-26. ISSN: 2038-9736

Scriven M. (1999). Evaluation thesaurus. Newbury Park, CA: Sage.

Vallerand R.J., Pelletier L.G., Blais M.R., Briere N.M., Senecal C., Vallieres E.F. (1992). The Academic Motivation Scale: A measure of intrinsic, extrinsic and amotivation in education. Educational and Psychological Measurement, 52: 1003 1017. DOI: $10.1177 / 0013164492052004025$

Vertecchi B. (1976). Valutazione formativa. Torino: Loescher.

Walberg H.J., Haertel G.D., a cura di (1990). International encyclopedia of educational evaluation. Oxford - New York - Berlin - Frankfurt - São Paulo Sydney - Tokyo - Toronto: Pergamon Press.

Zimmerman B.J, Bandura A., Mattinez- Pons M. (1992). Self-Motivation for Academic Attainment: The Role of Self-Efficacy Beliefs and Personal Goal Setting. American Educational Research Journal, 29(3): 663-676. DOI: 10.2307/1163261 